УДК 537.6

\title{
Features of the Non-equilibrium Critical Dynamics in 3D Pure and Diluted Ising-like Ferromagnets
}

\author{
Vyacheslav V. Krizhanovskiy* \\ Peter N. Malyarenko ${ }^{\dagger}$ \\ Pavel V. Prudnikov $\ddagger$ \\ Vladimir V. Prudnikov ${ }^{\S}$ \\ Department of Theoretical Physics \\ Dostoevsky Omsk State University \\ Mira, 55a, Omsk, 644077
}

Russia

Received 20.08.2016, received in revised form 10.10.2016, accepted 12.11.2016

The non-equilibrium critical behaviour of the three-dimensional pure and site-diluted spin systems described by Ising model is studied with different spin concentrations for evolution from various initial magnetic states. The universal scaling functions are determined for the magnetization and autocorrelation function. The aging and superaging effects are revealed in autocorrelation function behaviour for diluted systems with evolution from low-temperature initial states.

Keywords: non-equilibrium critical behaviour, Ising model, initial state, structure defects, correlation function.

DOI: 10.17516/1997-1397-2016-9-4-463-468.

\section{Introduction}

The investigation of critical behaviour of disordered systems remains one of the main problems in condensed-matter physics and excites a great interest because all real solids contain structural defects. The structural disorder breaks the translational symmetry of the crystal and thus greatly complicates the theoretical description of the material. Then, to describe the influence of disorder on the critical behaviour the methods of computer calculations are used from which Monte-Carlo methods are main. The development of computer technology and increase of their capacity contributes to this direction of investigations.

Critical dynamics provides a simple instance of slow collective evolution. Statistical systems near the critical temperature are displayed by dramatic slowing down of relaxation processes, hysteresis, memory effects and etc. The influence of disorder is particularly important near critical point $T_{c}$ where the behaviour of a system is characterized by an anomalous large relaxation time described by the law $t_{r e l} \sim\left|T-T_{c}\right|^{-z \nu}$, where $z$ is the dynamic critical exponent and $\nu$ is the correlation length exponent. At a time $t \ll t_{r e l}$, the effects of aging manifest themselves, namely, at the two-time correlation and response functions on the waiting time $t_{w}$ and observation time $t-t_{w}$. An effect of initial states influence on the non-equilibrium critical behaviour is displayed within the $t, t_{w} \ll t_{r e l}$ time range $[1,2]$.

\footnotetext{
*FehtunKrizh@gmail.com

†petr.malyarenko@yandex.ru

${ }^{\ddagger}$ prudnikp@univer.omsk.su

§prudnikv@univer.omsk.su

(c) Siberian Federal University. All rights reserved
} 


\section{Characteristics of non-equilibrium critical behaviour and results of simulations}

In investigations of influence of the initial states on characteristics of non-equilibrium critical behaviour, there are differed the high-temperature initial state, when a system has been prepared at a temperature $T_{0} \gg T_{c}$ with the initial magnetization $m_{0}=0$ before it was quenched at $T_{c}$, and the low-temperature magnetized initial state with preparation at $T_{0}<T_{c}$ with $m_{0} \neq 0$. The non-equilibrium evolution starts when the system after initial preparation is placed to the thermostat with $T=T_{c}$. At the waiting time $t_{w}$, we have begun to measure two-time quantities such as response and correlation functions. We have considered the ferromagnetic Ising model site-diluted by nonmagnetic impurity atoms. The Hamiltonian of system is given by

$$
H=-J \sum_{<i, j>} p_{i} p_{j} S_{i} S_{j}
$$

where $J>0$ is short-range exchange interaction integral between spins $S_{i}= \pm 1$ fixed at the lattice sites. The random occupation numbers $p_{i}$ take the values 0 or 1 , and $p_{i}$ equals 1 if the site of the lattice contains spin and 0 otherwise. We considered the cubic lattice with periodic boundary conditions. Let us denote $N_{s}=p L^{3}$ as the number of spins in a lattice with linear size $L=64$ and 128 , where $p$ is the spin concentration.

To investigate the non-equilibrium evolution, we computed such characteristics as the magnetization $M(t)$ and the two-time dependent autocorrelation function $C\left(t, t_{w}\right)$, which are defined by relations

$$
\begin{aligned}
M(t) & =\frac{1}{V} \int d^{d} x[\langle S(x, t)\rangle]=\left[\left\langle\frac{1}{N_{s}} \sum_{i=1}^{N_{s}} p_{i} S_{i}(t)\right\rangle\right], \\
C\left(t, t_{w}\right) & =\frac{1}{V} \int d^{d} x\left[\left\langle S(x, t) S\left(x, t_{w}\right)\right\rangle-\langle S(x, t)\rangle\left\langle S\left(x, t_{w}\right)\right\rangle\right]
\end{aligned}
$$

where angle brackets stands for the mean over the different realizations of initial state, the square brackets are for averaging over the different impurity configurations.

At the present time, it becomes established that the time dependence for magnetization and autocorrelation function for systems starting from a low-temperature initial state with $m_{0} \neq 0$ satisfies the following scaling forms [1]:

$$
\begin{aligned}
M\left(t, t_{m}\right) & =A_{M} t^{-\beta / z \nu} F_{M}\left(t / t_{m}\right), \\
C\left(t, t_{w}, t_{m}\right) & =A_{C}\left(t-t_{w}\right)^{a+1-d / z}\left(t / t_{w}\right)^{\theta-1} F_{C}\left(t_{w} / t, t / t_{m}\right),
\end{aligned}
$$

where $t_{m}=B_{m} m_{0}^{-1 / k}$ is a new timescale with exponent $k=\theta^{\prime}+\beta /(\nu z)>0, a=(2-\eta-z) / z$, $\theta=\theta^{\prime}-a, \theta^{\prime}$ is the initial slip exponent. The scaling functions $F_{M}\left(t / t_{m}\right)$ and $F_{C}\left(t_{w} / t, t / t_{m}\right)$ are finite at limits $t_{w} \rightarrow 0$ and $t / t_{m} \rightarrow 0, A_{M}$ and $A_{C}$ are non-universal amplitudes.

At the beginning, we realized investigation of critical relaxation of magnetization $M(t)$ for pure $(p=1)$ and diluted systems $(p=0.8,0.6$, and 0.5$)$ with various initial states $m_{0}$ (Fig. 1a), which demonstrate that the relaxation time is increased with growth of the impurity concentration $c_{i m p}=1-p$. The curves in Fig. $1 \mathrm{~b}$ and $\mathrm{c}$ for pure and diluted systems with $p=0.6$ demonstrate an essential differences in relaxation from the high-temperature with $m_{0} \ll 1$ and low-temperature with $m_{0}=1$ initial states, and from the intermediate initial states with $0.2 \leqslant m_{0} \leqslant 0.6$. So, a remarkable property of the non-equilibrium critical relaxation from the high-temperature initial state is the increase of magnetization $M(t) \sim t^{\theta^{\prime}}$ with $\theta^{\prime}>0$. The initial rise of magnetization is changed to the well known decay $M(t) \sim t^{-\beta / z \nu}$ for $t>t_{c r} \sim m_{0}^{-1 / k}$. The critical relaxation from the completely ordered initial state with $m_{0}=1$ is immediately 
characterized by power-law of magnetization decay $M(t) \sim t^{-\beta / z \nu}$. The intermediate cases with $0.2 \leqslant m_{0} \leqslant 0.6$ and 0.4 are characterized by a short stage of magnetization rise under the powerlaw $M(t) \sim t^{\theta^{\prime}}$ which is changed to much long-continued stage of relaxation with $M(t) \sim t^{-\beta / z \nu}$.
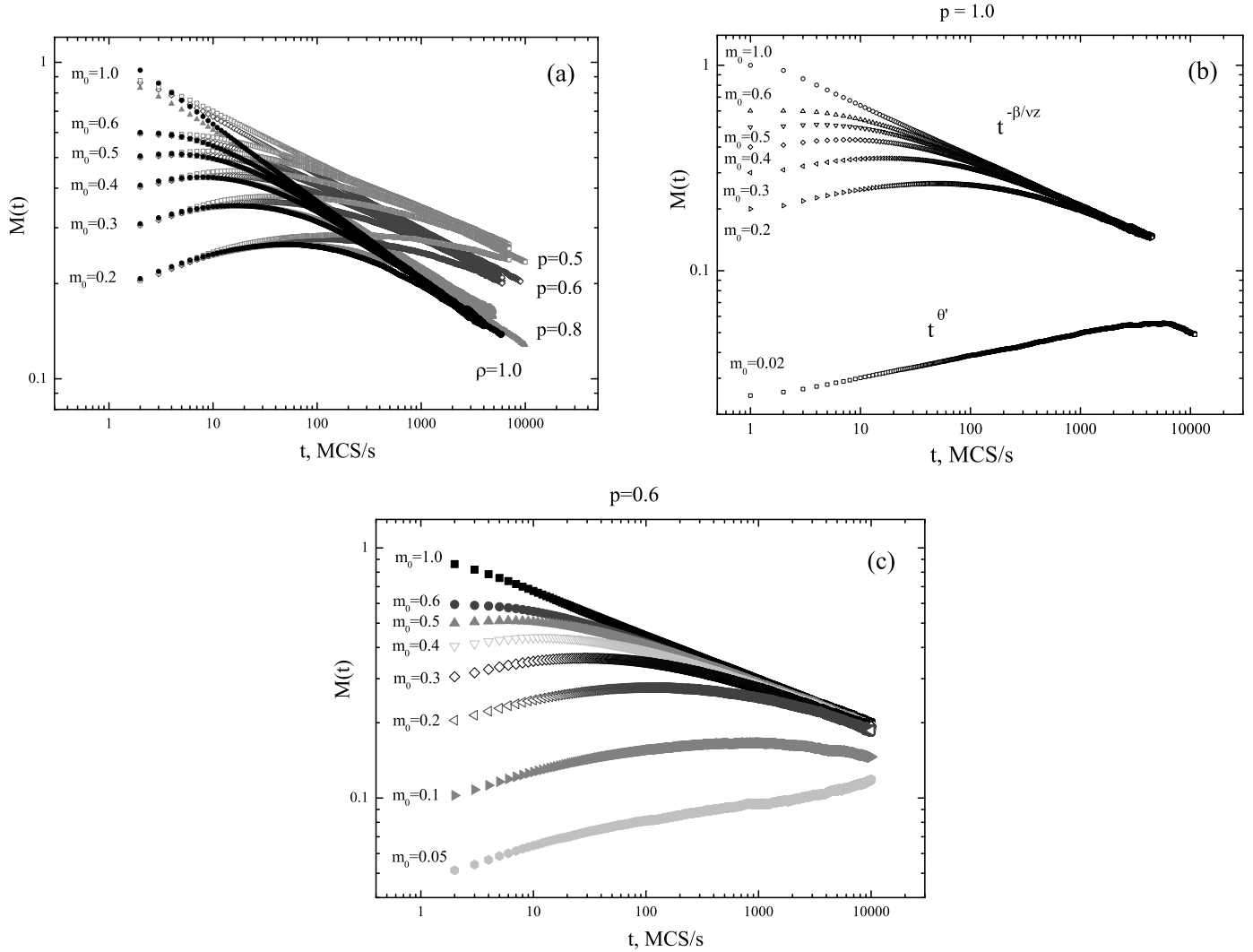

Fig. 1. Time dependence of the magnetization $M(t)$ for various values of the initial magnetization $m_{0}$ and different spin concentrations $p(\mathrm{a})$, for pure model, $p=1.0(\mathrm{~b})$ and diluted system, $p=0.6(\mathrm{c})$

To check the scaling prediction for $M(t)$ as function of the initial magnetization $m_{0}$ given by relation (3), we plot the dependence of $t^{\beta / z \nu} M(t)$ versus $x=t m_{0}^{1 / k}$ in Fig. 2 with the use of exponent values for the pure 3D Ising model: $\beta / \nu=0.516(2), z=2.024(6)$, and diluted systems: $\beta / \nu=0.508(16), z=2.191(21)$ for $p=0.8$ and $\beta / \nu=0.462(40), z=2.663(30)$ for $p=0.6$ [3]. We can see in Fig. 2a, b and c the collapse of curves $F_{M}(x)$ for different $m_{0}$ with fixed spin concentration $p$ into a single universal curve with linear initial curve piece (in $\log -\log$ scale) with $F_{M}(x) \sim x^{k}$. We obtained the following values of exponent $k$ : for pure case $k=0.361(3)$, for systems with $p=0.8 k=0.353(4)$ and with $p=0.6 k=0.325(7)$.

The dependence of the autocorrelation function on the initial magnetization $m_{0}$ in concordance with relation (3) can be determined when the waiting time is taken as a quantity proportional to the observation time, as example, $t_{w}=t / 3$. Then, the scaling dependence for the autocorrelation function in (3) takes on form:

$$
C\left(t, t_{w}=t / 3, t_{m}\right) \sim t^{-2 \beta / z \nu} G_{C}\left(t m_{0}^{1 / k}\right) .
$$

We presented in Fig. 3a and $\mathrm{b}$ the time dependence of function $C\left(t, t_{w}=t / 3, t_{m}\right)$ for pure and diluted with $p=0.8$ models for various values of the initial magnetization $m_{0}$, and in Fig. $3 \mathrm{c}$ 

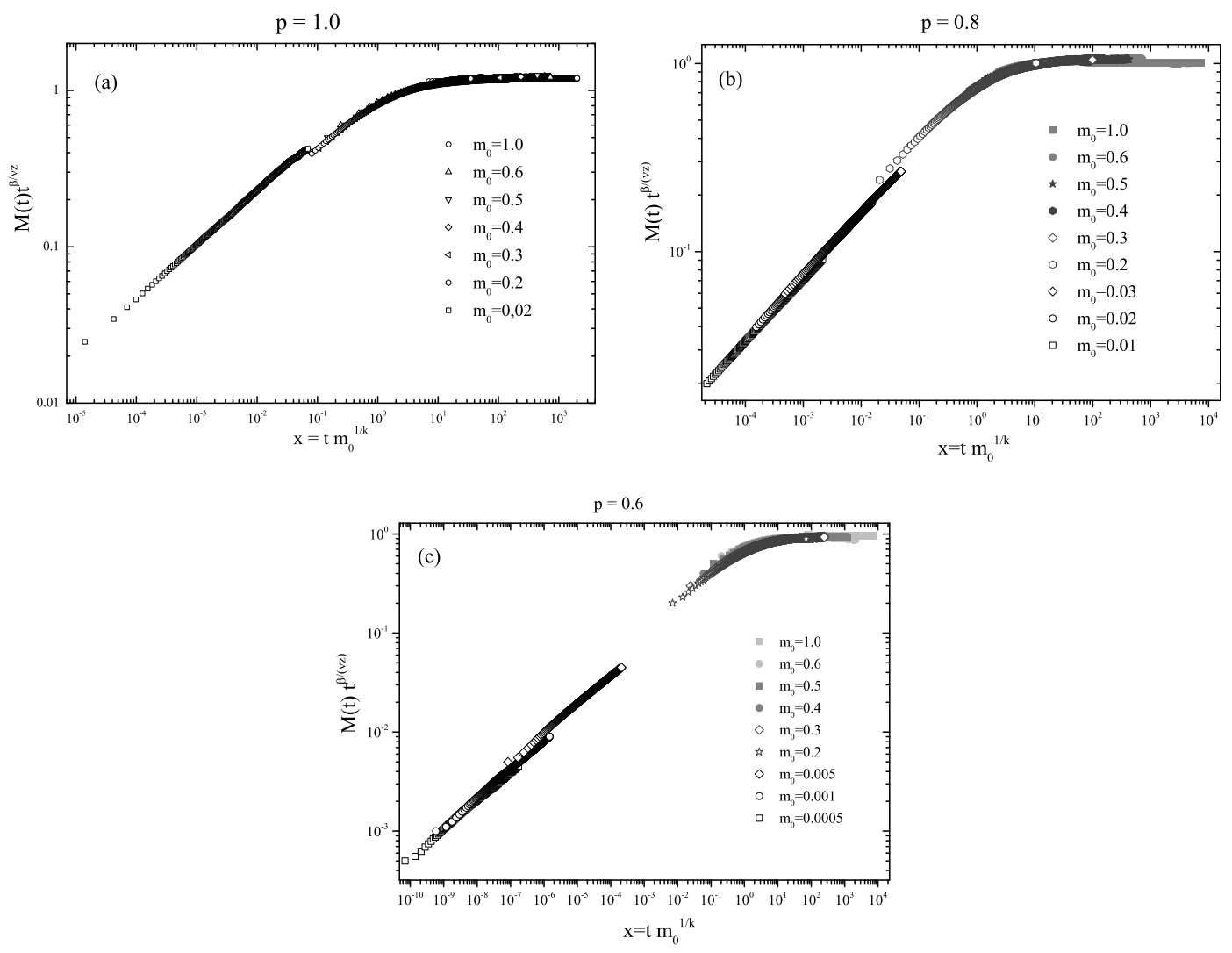

Fig. 2. Dependence of the magnetization scaling functions $F_{M}\left(t / t_{m}\right)=M(t) t^{\beta / z \nu}$ on $x=t m_{0}^{1 / k}$ for different initial magnetizations $m_{0}$ and spin concentrations $p=1.0$ (a), $p=0.8$ (b) and $p=0.6(\mathrm{c})$

and $\mathrm{d}$ the dependence of the scaling functions $G_{C}\left(t m_{0}^{1 / k}\right)=t^{2 \beta / z \nu} C\left(t, t_{w}=t / 3, t_{m}\right)$ on variable $x=t m_{0}^{1 / k}$ with $k=0.361$ for pure model and $k=0.353$ for system with $p=0.8$. The curves in Fig. $3 \mathrm{a}$ and $\mathrm{b}$ demonstrate the aging effects through deflection of the curves for time dependence of $C(t, t / 3)$ from power law, which must look as straight lines at log-log scale, and characterize the slowing down of time correlations with increasing system age $t_{w} \sim t$. One can see that the increase of $m_{0}$ leads to the amplification of aging effects.

The graph in Fig. 3c demonstrates the scaling collapse of function $G_{C}(x)$ for various values of $m_{0}$ into an universal curve that confirms the scaling forms (3) and (4) for pure Ising model. However, the graph in Fig. 3d shows the violation of scaling form (4) for lack of the data collapse for dilute system. We connect this phenomenon with essential influence of defects on nonequilibrium dynamics of magnetic domain structure and therefore on non-equilibrium correlation properties of diluted system, which lead to superaging effects [2]. When we use the presentation of autocorrelation function in the form $t^{2 \beta / \mu z \nu} C\left(t, t^{1 / \mu}, t_{m}\right)$ on $x=t^{\mu} m_{0}^{1 / k}$ it gives possibility to get with the use of superaging exponent $\mu=2.30(6)(p=0.8)$ [2] the data collapse into a single curve for values of the initial magnetization $m_{0}>0.25$ (Fig. 3f). Thus, the autocorrelation function is characterized in the aging regime with $t \geqslant t_{w}^{\mu}$ by more complicated scaling dependence in the form $C\left(t, t_{w}, t_{m}\right) \sim t_{w}^{-2 \beta / z \nu} \tilde{F}_{C}\left(t / t_{w}^{\mu}, t / t_{m}^{1 / \mu}\right)$ for the site-diluted Ising model.

Investigations were supported by Russian Science Foundation, project 14-12-00562. 

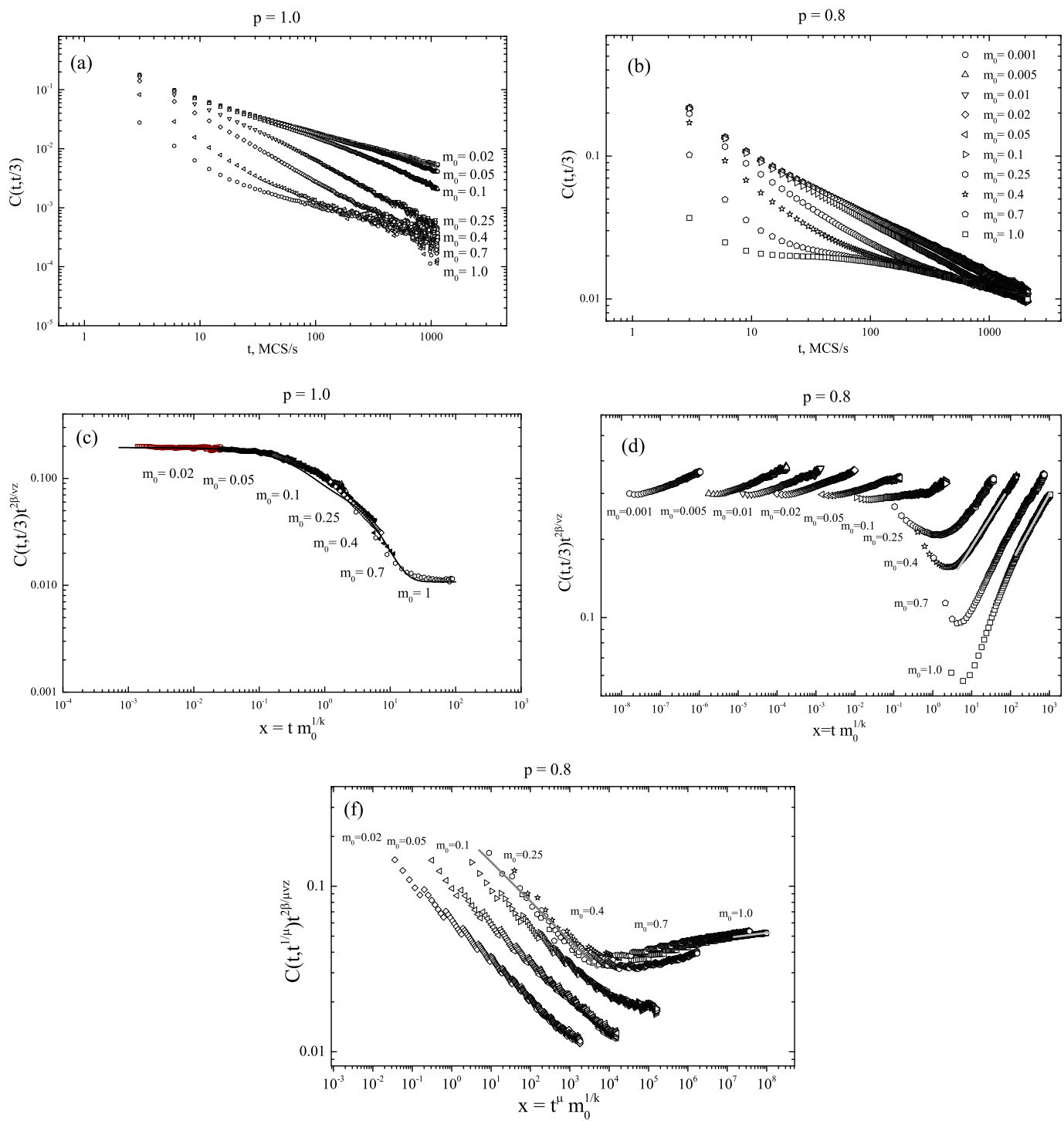

Fig. 3. Dependence of the autocorrelation function $C\left(t, t_{w}=t / 3, t_{m}\right)$ on time $t$ with different initial magnetizations $m_{0}$ for systems with $p=1.0$ (a) and $p=0.8$ (b) and its scaling functions $G_{C}\left(t / t_{m}\right)=C\left(t, t_{w}=t / 3, t_{m}\right) t^{2 \beta / z \nu}$ on $x=t m_{0}^{1 / k}$ for systems with $p=1.0$ (c) and $p=0.8$ (d). Superaging effects for scaling function $t^{2 \beta / \mu z \nu} C\left(t, t^{1 / \mu}, t_{m}\right)$ on $x=t^{\mu} m_{0}^{1 / k}$ for system with $p=0.8$ (f)

\section{References}

[1] P.Calabrese, A.Gambassi, F.Krzakala, J. Stat. Mech., (2006), P06016.

[2] V.V.Prudnikov, P.V.Prudnikov, E.A.Pospelov, J. Stat. Mech., (2016), 043303.

[3] P.V.Prudnikov, V.V.Prudnikov, E.A.Pospelov, P.N. Malyarenko, A.N.Vakilov, Prog. Theor. Exp. Phys., (2015), 053A01. 


\title{
Особенности неравновесной критической динамики в чистых и разбавленных трехмерных изингоподобных ферромагнетиках
}

\author{
Вячеслав В. Крижановский \\ Петр Н. Маляренко \\ Павел В. Прудников \\ Владимир В. Прудников \\ Физический факультет \\ Омского государственного университета \\ Мира, 55а, Омск, 644077 \\ Россия
}

\begin{abstract}
Представлены результаты численного исследования особенностей неравновесного критического поведения в трехмерной чистой и структурно неупорядоченной модели Изинга с широким спектром изменения спиновой концентрачии при её эволючии из различных начальных магнитных состояний. Выявлены эффекты старения и сверхстарения в двухвременном поведении автокорреляционной функции для структурно неупорядоченных систем при эволючии из низкотемпературных начальных состояний.

Ключевые слова: неравновесное критическое поведение, модель Изинга, началъное состояние, дефекты структуры, корреляционная функиия.
\end{abstract}

